\title{
Permeability rules for antibiotic design
}

Failure seems to be the buzzword in antibiotics research. Vast synthetic molecule libraries are devoid of new antibiotics, and foraging through the so-called 'genomic dark matter' of uncultivable bacteria using increasingly sophisticated sequencing technologies has not delivered therapeutic antibiotic candidates. With antibiotic resistance acknowledged as a crisis by the World Health Organization in 2015, we sorely need to find ways to replenish the antibiotics pipeline. Now, chemist Paul Hergenrother, Michelle Richter and their colleagues from the University of Illinois at Urbana-Champaign have devised a strategy to reveal features of small molecules that make them likely to accumulate inside Gramnegative bacteria. They applied their findings to convert an antibiotic that only kills Grampositive bacteria into a broad-spectrum agent capable of also killing Gram-negative bacteria and report their results in Nature ${ }^{1}$.

The Gram stain, a simple, quick and cheap stain (crystal violet) for detecting bacterial peptidoglycan, is a mainstay of clinical microbiology. Bacteria can be differentiated into two groups using this stain: Gram-positive bacteria, which have a single outer membrane and a cell wall, and Gram-negative bacteria, which have an inner membrane, a cell wall and an outer membrane. Some antibiotics target either one or the other of these types. Antibiotics need to get inside bacterial cells to kill them, but for Gram-negative bacteria this is not as simple as it sounds. "Gram-negative bacteria are more difficult to kill because the outer membrane poses an additional barrier to molecule entry," Hergenrother explains. Notable Gramnegative pathogens include Escherichia coli and Pseudomonas aeruginosa.

Decades of painstaking research to discern how antibiotics enter and exit cells have revealed that molecules that kill Gramnegative bacteria are likely to be very polar and less than $600 \mathrm{Da}$, but this doesn't explain why many molecules fitting this description are not antibiotics. Perhaps if research had been focused on how antibiotics get into cells (rather than on how any molecule gets into cells), a bias toward finding certain molecular features that are only present in alreadydiscovered antibiotics might have occurred. Reasoning that a detailed understanding of which molecules can enter and accumulate in Gram-negative cells would best underpin

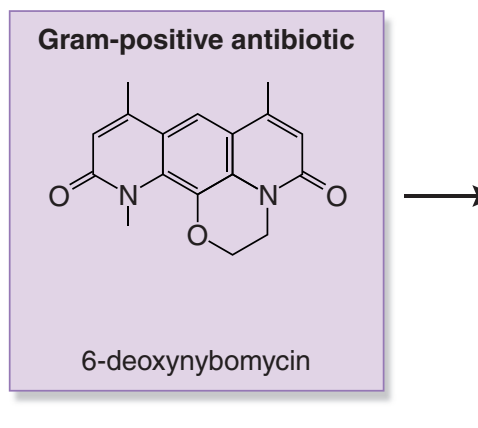

efforts to design antibiotic therapeutics, Richter and colleagues tested a panel of 100 molecules on the best-studied Gram-negative bacteria of them all, E. coli. These complex and diverse compounds were synthesized using a strategy called 'complexity-to-diversity', published by Hergenrother's lab in 2013 (ref. 2). The first surprise was that charge seemed to be the main feature that affected molecule accumulation, rather than size or polarity. Specifically, positively charged molecules accumulate inside cells, whereas neutral or negatively charged molecules do not.

Of the 12 compounds that accumulated inside E. coli, all contained an amine and eight were primary amines. Laura Piddock, at the University of Birmingham, comments that "although previous researchers, most notably Hiroshi Nikaido, and then others including my team, in the 1980s investigated the relationship between structural attributes and properties of antibiotics, I was unaware of the benefit of ionizable nitrogen [in the antibiotic molecule] in drug accumulation within the bacterial cell." The next step for Richter and colleagues was to use a wider range of primary amines in accumulation experiments, to understand why some of the primary amines in the initial collection accumulated, whereas others failed to accumulate. The authors assessed a diverse panel of 68 additional primary aminecontaining compounds and found that 36 of these accumulated in E. coli.

They then calculated 297 molecular descriptors for each of the 36 amine-accumulators and for the 32 amine nonaccumulators (from the panel of 68 primary amines), and this data was used to train a random-forest classification model. According to the random-forest model outputs, the features of a molecule that is predicted to accumulate in E. coli are "a nonsterically encumbered amine, nonpolar functionality, rigidity and low globularity" ${ }^{\text {. }}$
Finally, Richter et al. ${ }^{1}$ surveyed a set of antibiotics that kill only Gram-positive bacteria and identified deoxynybomycin, a natural-product antibiotic that inhibits DNA gyrase, as a good candidate for conversion to a broad-spectrum antibiotic. By expanding the five-membered ring of deoxynybomycin to a six-membered ring, they were then able to add a primary amine. The resulting 6DNM-NH3 killed both E. coli and other Gram-negatives, as well as Staphylococcus aureus, a Gram-positive.

Although the synthetic broad spectrum agent didn't kill $P$. aeruginosa, Hergenrother is convinced that "the same blueprint of finding features that enable accumulation in cells and then using modeling to understand which of those features are likely to be crucial for accumulation will be successful for defining accumulation parameters for other pathogenic bacteria." Furthermore, he mentions that "lots of drugs have primary amines, including daptomycin and Tamiflu [oseltamivir], so toxicity will likely not prove problematic."

Piddock agrees that this approach has merit, saying, "The authors used their analysis to predict compounds that would accumulate within bacterial cells and synthesized exemplar compounds. Whilst the compounds they made are unlikely to be developed into drugs, this work will certainly help others (as well as themselves) discover compounds with the appropriate pharmacological properties, as well as the ability to accumulate to high concentrations within bacterial cells."

Susan Jones, Senior Editor

1. Richter, M.F. et al. Nature 545, 299-304 (2017).

2. Huigens, R.W. III et al. Nat. Chem. 5, 195-202 (2013). 